\title{
ECOPOL YTECHNIC: A SUSTAINABLE AND FLEXIBLE E-LEARNING SYSTEM FOR AGILE SMART LEARNING SCENARIOS
}

\author{
Michele Angelaccio \\ University of Rome "Tor Vergata" - Management Engineering Dept, Via del Politecnico 1, 00100 Rome, Italy
}

\begin{abstract}
The rapid change of technological and human-machine co-evolutionary processes due to smart working and smart industries, present several issues that are also influencing the world of e-learning systems. Nowadays is quite hard to distinguish between learning spaces/systems/organizations and working organizations especially when smart working and tele-monitoring are becoming widespread. In this work we analyze smart learning concepts that are leading to a new emerging scenario in which the distinction between learning and working activities is dynamic and in evolution. In particular we present an integrated smart learning model called EcoPolytechnic which aims to redesigns the organization model of a Polytechnic by introducing several concepts of agile learning and project-based learning. The model is built upon the notion of smart learning cell that replaces in a flexible and sustainable way the notion of set of courses and can be used in a better way in smart learning scenarios. The result is a complete and easy to be configured smart learning organization system for which a comparative analysis is carried out. The benefits in terms of innovation and adaptive learning performance are given by using agile learning analysis.
\end{abstract}

\section{KEYWORDS}

Data Science, Smart Learning, Agile Learning

\section{INTRODUCTION}

Technology is transforming jobs and skills faster than the ability of organizations or people to adapt to them. Coursera's Global Skills Index 2019 found that two-thirds of the world's population is falling behind in critical skills. Research from the World Economic Forum suggests that the core skills required to perform most roles will change by $42 \%$ on average within 2022 . At this level of disruption, companies are scrambling to identify and source the skills they need in order to stay competitive. The availability of key skills is now one of the top three business threats for CEOs globally, according to a recent PwC survey. Driven by these impulses, new scenario of learning systems (Figure 1) are emerging, in which online courses are required in several ways and at different places with the aim of yielding digital skills capabilities in an effective way and often as soon as possible. Moreover interdisciplinary approaches besides typical engineering sectors as for instance social science, are leading to consider a new knowledge base of social data science extending the role of scientific data science (see Lifelong learning schema in Figure 1- discussed in Piano Scuola Digital).

In this way, we can say that a new era of smart learning activities is becoming a reality. Unfortunately the traditional model of university campus with physical or virtual online learning activities carried out in a fixed place and with a long-range organization system, does not seem to satisfy well this change, because it hardly cope with dynamic digital skills required by several stack holders and smart industries (Kavasakalis \& Lioss, 2019; Angelaccio \& Buttarazzi, 2010 and Angelaccio \& Buttarazzi, 2016).

As noted in Sonetti, Lombardi, and Chelleri (2016), in order to obtain a new approach for green campuses, there is the need to propose "clusters of different university typologies", and to this purpose they remarked at micro-scale, the need for revising and replacing current eco-efficiency-driven indicators with more life cycle and user-centric related metrics. 


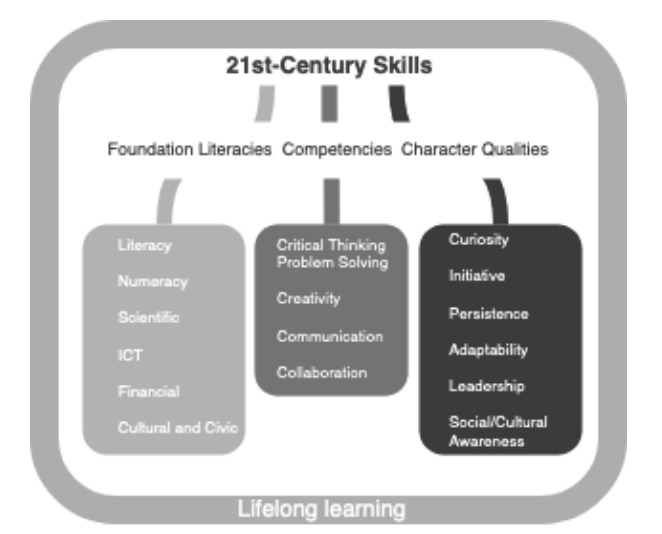

Figure 1. New Vision for Education (World Economic Forum 2013)

In this paper we try to explore this direction of new modeling with user-centric approach and outline a different approach for smart learning system more adequate to digital competencies and skills.

\section{ECOPOLYTECHNIC DESCRIPTION}

\subsection{Smart Learning Concepts and EcoPolytechnic Organization}

Typical Engineering Learning Activities performed in a so-called Polytechnic System, are characterized by Multiple Engineering Sectors shared among the same students and researchers with the aim to be very interdisciplinary.

Figure 2 shows the traditional organization schema of a campus performing learning and research activities in synergy with external communities by focusing on the smart city context (Polytechnic organization and smart city).

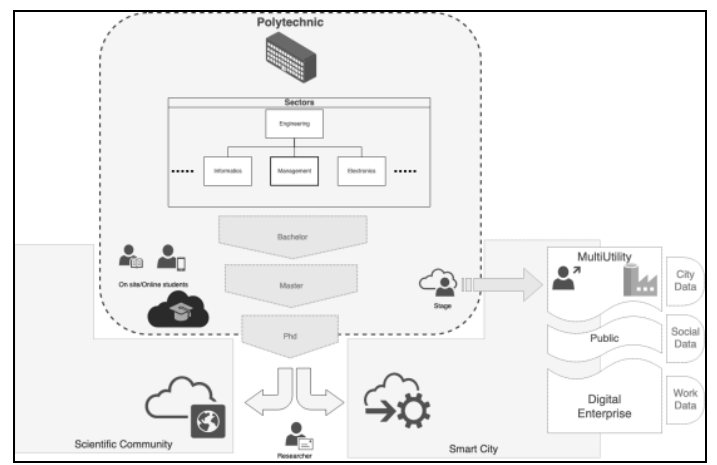

Figure 2. Traditional Polytechnic organization schema

To overcome this organization scheme and provide a different organization for e-learning in a smart scenario, we introduce a smart learning organization built upon a set of hybrid educational/coworking islands where it will be possible to learn and apply digital technologies. These are called smart learning cells (SLC) and allow an easy configuration in several places and in several ways, with little organizing effort. Basically they are composed by web notebooks working on datasets incoming from real environment (IoT datasets for example) and targeted to particular learning outputs matching some of the real needs. Each cell acts as a minimal learning space involving a working group assigned to a data project related to a smart city subfield. Tutor starts the activities, but after an initial scrum period the SLC will proceed autonomously and will be monitored independently. After a minimal learning activity, the cell will output a set of digital results hosted by the cloud lab platform. The main novelty, compared to previous models, concerns the fact that the 
resulting learning system works as a virtual organization box that could be embedded in a real scenario depending on the original dataset and the corresponding set of learning objectives, without the need of a particular physical space as a Campus or University Building. This is the main reason for the choice of the eco prefix label used in the Organization Model name presented here, and it must to be considered as a further step beyond well-known concepts used in e-learning technologies (online course, webinar, etc.).

In particular, there is a need to reinforce the motivation of a new Model based on a more agile learning phases obtained via optimization of staging and online courses better suited to industry and working needs.

To this purpose, it occurs to change the overall process to reach a more flexible and dynamic organization level, in which small learning units are definable in a suitable way and customizable to different working needs in an easy way but at the same time by ensuring a minimal knowledge base in the learning curricula.

Figure 3 gives an example of learning organization that could be implemented by a different Polytechnic system called eco-Polytechnic in which the organization is based on cells (subfigure (a)), called Smart Learning Cell (SLC) in which, through the use of notebooks, agile learning with data science paradigms yields a scrum process that monitors learning activity.

In this way the learning organization could be reconfigured on the basis of learning goals and depending on the external scientific data and corresponding working context (subfigure (b)). This adaptability to the context and the capability for a single cell to be implemented in a flexible way both in a campus or in other place will introduce the novel schema and its property to be eco-sustainable in the sense that it works in accord to smart working and smart learning objectives.

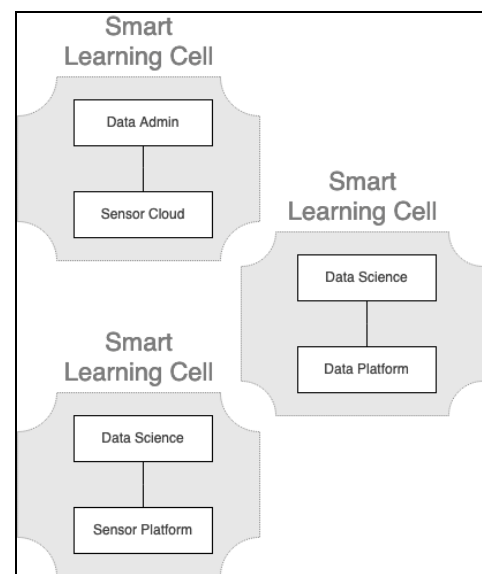

(a)

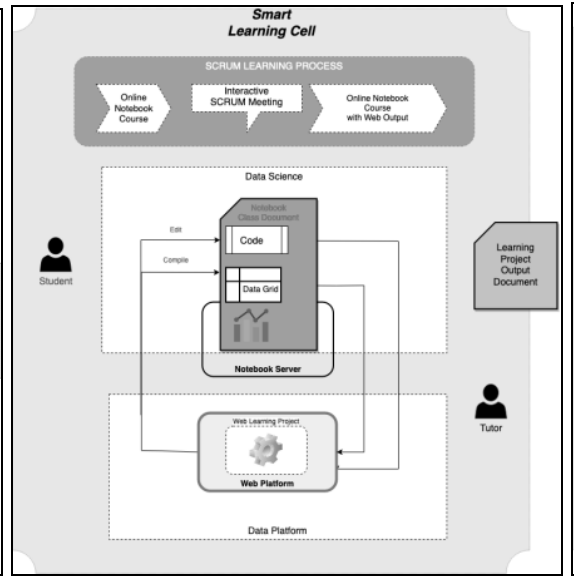

(b)

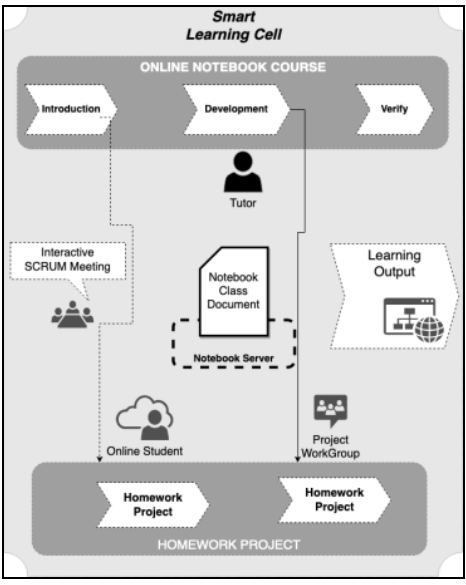

(c)

Figure 3. EcoPolytechnic organization schema: (a) Smart Learning Cells, (b) SLC Description, (c) SLC Workflow

The data science topics are organized in two main blocks (Content and Platform) by using the notion of web notebook with interactive learning sections useful to learn in an autonomously and flexible way all digital foundations and using real data example downloaded by internet.

In this way active learning becomes a reality in data science course through notebooks due to the following main reasons:

- Notebooks can be used without requiring particular preliminaries on digital skills that could be easily integrated in the same notebook.

- Learning output are easily managed without the need of a different web learning platform

- Agile development of learning output and results for certifying digital skills could be easily deployed in terms of web platforms described through notebook code examples with digital repositories.

In this way we obtain a small organization learning box system which is more flexible and suitable to be used in a cellular based learning system thanks to the capability to be connected in several way to obtain a chain of Smart learning Cells for Smart learning Scenarios (see Workflow description in (subfigure (b)).

In the next section we give some example of smart learning thrugh SLC, to evidence the capability to accelerate learning outputs in interdisciplinary socio technological sectors like Smartourism and Smart City. 


\subsection{Smart Learning Scenarios Examples}

Digital Skills for Smart City technologies often require an interdisciplinary approach for which a flexible and dynamic e-learning approach must kept into account (Smart Learning Scenarios). In these cases it could be useful to focus on project-based learning designed on the basis of common data science topics since public open data are the common source of required digital skills and competencies for which there is the main attention by stakeholders (Kavasakalis, \& Lioss, 2019).

This activity is quite independent from other learning activities and can be executed in a project-based framework without the need to be considered simply as a set of lessons.

The required learning re-organization will put research and students at the nexus of neighborhood networks (see Figure 4). EcoPolytechnic could be used to engage students in external community and enterprises having a common data science paradigm.

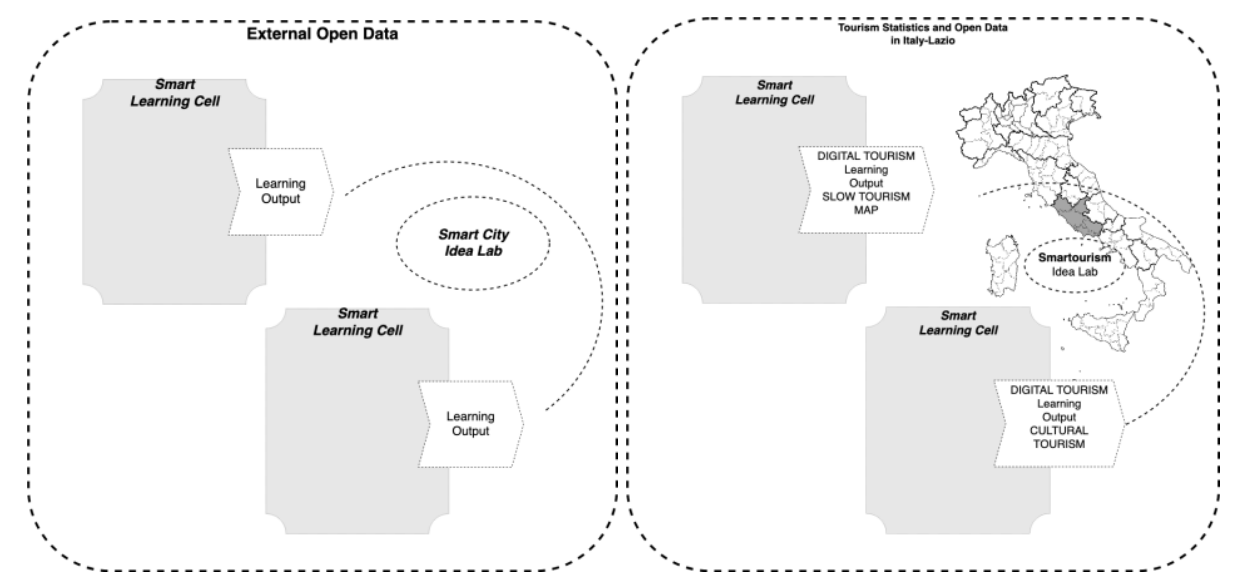

Figure 4. EcoPolytechnic Cellular Organization for Smart City data science

As example of this interaction, Figure 4 (on the right) outlines the case of smart learning scenario resulting by the application of a two learning cells designed to approach data science for smartourism for which the referenced data are taken by touristic places aroud Rome in Italy (Lazio Region).

\section{REFERENCES}

Angelaccio, M. \& Buttarazzi, B. (2016) Enhancing web programming learning through mobile ecommerce paradigms in atti di Didamatica 2016, Udine, 19-21 aprile 2016, ISBN: 978889809144.

Angelaccio, M. \& Fanti, A. (2011), Design of active learning strategy through agile development methodologies: a technological view, in: CECIIS-2011.

Angelaccio, M. \& Buttarazzi, B. (2010) A social network based-enhanced learning system, in: S. Reddy (Ed.), 19th IEEE International Workshopson Enabling Technologies: Infrastructures for Collaborative Enterprises, WETICE 2010, Larissa, Greece, 28-30 June 2010, Proceedings, IEEEComputer Society, 2010, pp. 94-95.

Kavasakalis, A. \& Lioss, F. (2019) Lifelong Learning Policies: The Case of Work-Based Learning, July 2019 DOI:10.5296/jet.v6i2.14804 Journal of Education and Training ISSN 2330-9709 2019, Vol.6, No.2

Piano Scuola Digitale (Italian Digital School Program) https://www.miur.gov.it/scuola-digitale

Sonetti, G., Lombardi, P. \& Chelleri, L. (2016). True Green and Sustainable University Campuses? Toward a Clusters Approach. in Sustainability 8(1):83 January 2016.

Taylor, K \& Negron, R \& Bell, C \& Riesland, A \& Taylor, Katie \& Silvis, Deborah \& Cramer, C.. (2019). Supporting Public-Facing Education for Youth: Spreading (Not Scaling) Ways to Learn Data Science With Mobile and Geospatial Technologies 